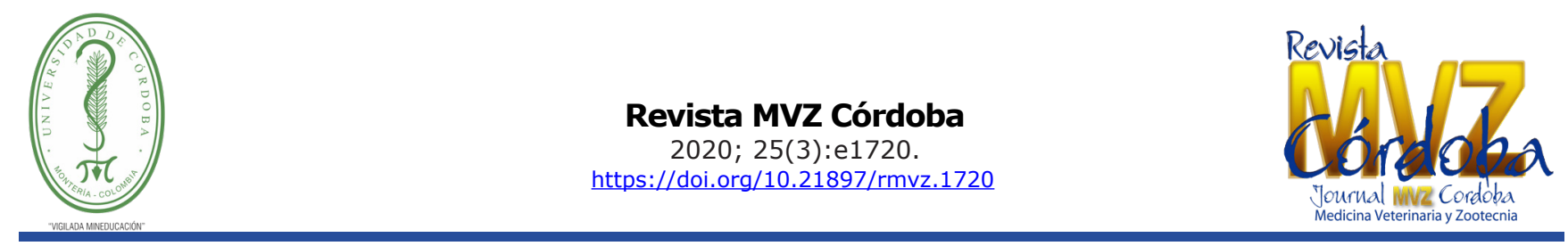

Original

\title{
Caracterización espacial de la ganadería bovina en la Orinoquia colombiana
}

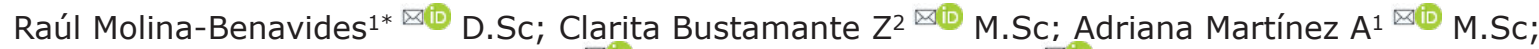 \\ José Uribe $C^{1} \bowtie\left(\mathbb{0}\right.$ M.Sc; Johan Redondo $\mathrm{O}^{2} \bowtie$ Ph.D.
}

${ }^{1}$ Universidad Nacional de Colombia sede Palmira. Carrera 32 \# 12-00, Palmira-Colombia.

${ }^{2}$ Instituto de investigación de recursos biológicos Alexander von Humboldt. Calle 28A \# 15-09, Bogotá D.C - Colombia.

*Correspondencia: ramolinab@unal.edu.co

Recibido: Junio 2019; Aceptado: Abril 2020; Publicado: Agosto 2020.

\section{RESUMEN}

Objetivo. Utilizar los sistemas de información geográfica (SIG) como herramienta complementaria para caracterizar la ganadería bovina realizada en la región de la Orinoquia. Materiales y métodos. A través del uso de tecnologías espaciales se recopiló la información concerniente a la orientación ganadera, fisiografía, cobertura vegetal y catastro de la zona de estudio para su posterior análisis a través del software ACCESS de Microsoft. Resultados. En un alto porcentaje de los predios ganaderos ubicados en los cuatro departamentos de la Orinoquía (Casanare:72.7\%, Meta:49.5\%, Arauca:42\% y Vichada:32\%) predominan las coberturas de pastos, herbazales y vegetación secundaria, confirmando la expansión en la frontera agropecuaria que es promovida por la actividad ganadera en el país. Conclusiones. El uso de los SIG, permite realizar una mejor planificación y distribución eficiente de los recursos destinados a mejorar el funcionamiento de los sistemas de producción. Por ejemplo, en zonas donde la matriz de coberturas predominante son los pastizales y herbazales, las estrategias en pro de la sostenibilidad pueden enfocarse en la implementación de sistemas silvopastoriles, contrario a lo que pasaría en zonas donde la matriz de coberturas tenga un alto porcentaje de bosques naturales.

Palabras clave: Análisis de datos espaciales; coberturas de la tierra; sistemas de información geográfica; polígonos (Fuente: $C A B$ ).

\section{ABSTRACT}

Objective. Use Geographic Information Systems (GIS) as a complementary tool to characterize cattle farming in the Orinoquia region. Materials and methods. Through the use of space technologys, information concerning the livestock orientation, physiography, vegetation cover and land registry of the study zone was collected for further analysis over Microsoft ACCESS software. Results. In a high percentage of the cattle ranches located in the four departments (Casanare: $72.7 \%$, Meta: $49.5 \%$, Arauca: $42 \%$ and Vichada: $32 \%$ ) the cover of pastures, grasslands and secondary vegetation predominates, confirming the expansion in the agricultural border that has had the cattle activity in the country. Conclusions. The use of complementary tools such as GIS allows for better planning and efficient distribution of resources to improve the functioning of production systems, for example,

Como citar (Vancouver).

Molina BR, Bustamante ZC, Martínez A, Uribe CJ, Redondo OJ. Caracterización espacial de la ganadería bovina en la Orinoquia colombiana. Rev MVZ Córdoba. 2020 25(3):e1720. https://doi.org/10.21897/rmvz.1720 CC) 5 CEl (los) autor (es), Revista MVZ Córdoba 2020. Este artículo se distribuye bajo los términos de la licencia internacional Creative Commons Attribution 4.0 (https://creativecommons.org/licenses/by-nc-sa/4.0/), que permite a otros distribuir, remezclar, retocar, y crear a partir de su obra de modo no BY NC SA comercial, siempre y cuando den crédito y licencien sus nuevas creaciones bajo las mismas condiciones. 
in zones where the predominant coverage matrix is grasslands, strategies in pro of sustainability can focus on the implementation of silvopastoral systems, contrary to what would happen in areas where the matrix has a high percentage of natural forests.

Keywords: geographic information systems; land cover; polygons; spatial data analysis. (Source: $C A B)$.

\section{INTRODUCCIÓN}

Aproximadamente un $40 \%$ de la tierra arable global está dedicada a la producción ganadera, lo cual se materializa en grandes extensiones de tierra ocupadas por pasturas y cultivos para la alimentación de estos animales $(1,2,3)$. Colombia no ha sido la excepción en cuanto el uso de su área continental, la ganadería está usando un $85 \%$ más de tierras sin vocación para esta actividad, trayendo consigo problemas de conservación y manejo inadecuado del medio ambiente $(4,5,6)$.

En Colombia, más del $95 \%$ de los sistemas de producción se basan en el pastoreo (7), es decir, los animales y su entorno están expuestos directamente al ambiente, generando efectos, muchas veces, de realimentación negativa que resultan en la degradación de los sistemas productivos y los recursos naturales que los sustentan $(7,8)$.

Colombia posee un inventario bovino cercano a los 23 millones de animales, los cuales se ubican en 39 millones de hectáreas, clasificando la actividad ganadera del país como extensiva. Esta carga animal de 0.6 animales/ha no ha variado significativamente en los últimos veinte años, revelando la baja transformación tecnológica del sector ganadero $(8,9)$.

Del inventario total, el $56 \%$ son hembras, $26 \%$ son machos y el restante son animales menores de un año; esta actividad se desarrolla en 500.000 fincas. Grosso modo, la actividad ganadera colombiana tiene tres orientaciones: i) producción de carne, ii) producción de leche y iii) doble propósito. Solamente el 5\% del inventario nacional es clasificado como lechería especializada, mientras que el $35 \%$ se dedica al doble propósito y el $60 \%$ restante se ocupa de las actividades de ceba y cría en forma extensiva $(8,9,10)$.

De los 23 millones de cabezas de ganado bovino presente en Colombia para el año 2015, el 21.52\% se encontraba en la región de la Orinoquia (según el inventario ganadero reportado por el ICA). Tradicionalmente, esta región se ha caracterizado por una ganadería extensiva dedicada a la producción de carne, exhibiendo una orientación del $50 \%$ para carne, $40 \%$ doble propósito y $10 \%$ leche $(11,12)$. Históricamente, los departamentos de Casanare y Meta han aportado el mayor número de animales al inventario de la región, seguidos por los departamentos de Arauca y Vichada (Figura 1).

\section{INVENTARIO GANADERO HISTÓRICO ORINOQUIA}

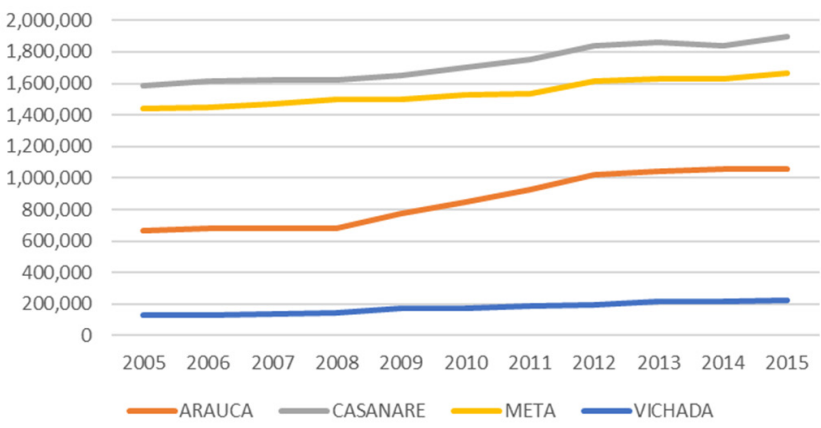

Figura 1. Inventario ganadero histórico 2005-2015 de la región de la Orinoquia. Fuente: ICA.

Dada la complejidad de los agroecosistemas ganaderos debido a sus múltiples componentes (suelo, cobertura vegetal, relieve, agua, entre otros) (4), se hace necesario recurrir a metodologías que permitan conocer y entender la dinámica de los sistemas productivos $(4,8,13)$.

Aparece entonces la caracterización biofísica como herramienta válida a la hora de conocer cómo se encuentran conformados los sistemas ganaderos; cuáles son sus componentes bióticos, limitantes y potencialidades respecto a otros sistemas (14), en busca de generar políticas que promuevan la sostenibilidad de estos agroecosistemas $(4,8,15)$.

Esta caracterización puede ser abordada a través de tecnologías novedosas como son los sistemas de información geográfica (SIG), los cuales permiten 
estudiar y analizar agroecosistemas en diferentes escalas espaciales para generar información más precisa sobre su uso y manejo (16).

Por lo anteriormente expuesto y cambiando el paradigma de que la ganadería en Colombia se puede clasificar solamente por su orientación, el número de cabezas por unidad de superficie, el uso de insumos y la introducción de tecnología, desconociendo las diversas combinaciones de los factores antes mencionados y las diferentes coberturas vegetales y relieves propios del territorio colombiano, que pueden incidir en las dinámicas de la actividad ganadera, el objetivo de este documento es caracterizar la ganadería bovina realizada en la región de la Orinoquia colombiana de acuerdo al número de predios encontrados bajo las diferentes orientaciones ganaderas, fisiografías y coberturas vegetales, por medio de SIG como herramienta de caracterización complementaria.

\section{MATERIALES Y METODOS}

Localización. La caracterización de la ganadería bovina realizada en la región de la Orinoquia (Figura 2), se realizó siguiendo los pasos propuestos Bermúdez et al (6) y Ochoa y Valerazo (17). A través de SIG, se recopiló cartografía base de parámetros geográficos como: orientación ganadera, fisiografía, cobertura vegetal y catastro de la zona de estudio

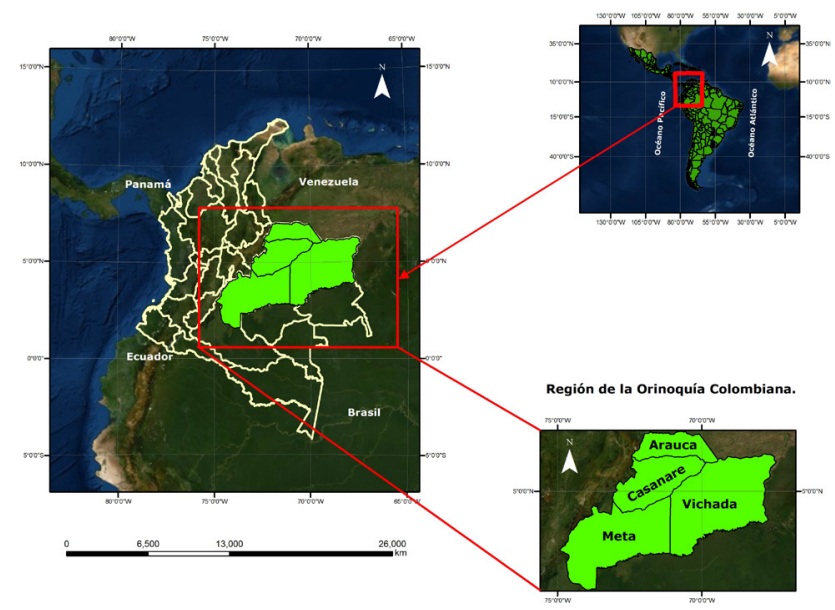

Figura 2. Área de estudio correspondiente a la región de la Orinoquia colombiana. Fuente: elaboración propia.

Fisiografía de la región de la Orinoquia. Las formas del relieve, identificadas y definidas a partir del análisis integral de la información topográfica, geológica, hidrológica y edafológica, permiten formar unidades relativamente homogéneas para su análisis (18).

Para la región de la Orinoquia se encontraron ocho superficies terrestres homogéneas (Figura $3)$, las cuales se nombran a continuación:

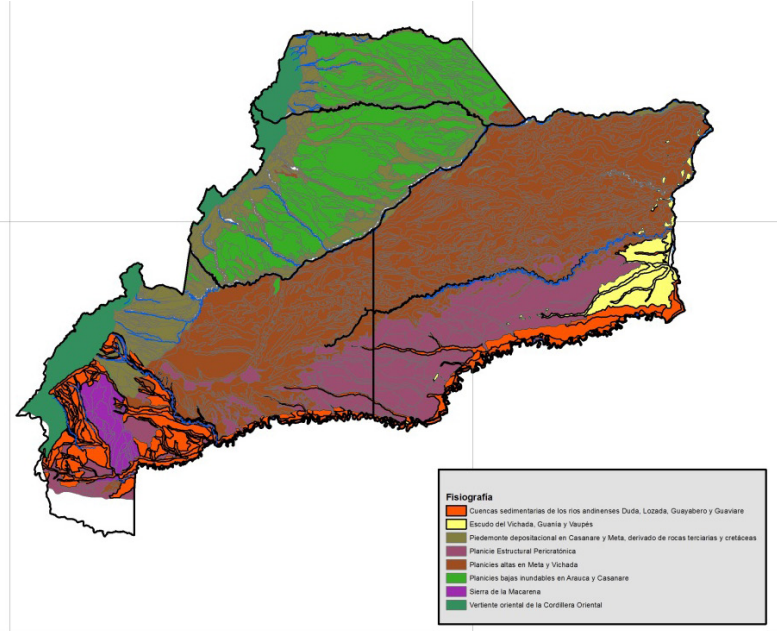

Figura 3. Relieves encontrados en la Orinoquia colombiana.

Fuente: Instituto de investigaciones Biológicas Alexander von Humboldt.

Cuencas sedimentarias de los rios andinenses Duda, Lozada, Guayabero y Guaviare (CSRADLGG).

Escudo del Vichada, Guainía y Vaupés (EVGV). Piedemonte depositacional en Casanare y Meta, derivado de rocas terciarias y cretáceas (PDCM). Planicie Estructural Pericratónica (PEP).

Planicies altas en Meta y Vichada (PAMV).

Planicies bajas inundables en Arauca y Casanare (PBIAC).

Sierra de la Macarena (SM).

Vertiente oriental de la Cordillera Oriental (VOCO).

En la Figura 3, se puede observar la distribución fisiográfica de la región de la Orinoquia. Este mapa se utilizó para el análisis de la intersección entre la orientación ganadera y su ubicación dentro del relieve del área de estudio.

Coberturas vegetales de la región de la Orinoquia. La asociación espacio-temporal de elementos biológicos vegetales característicos, los cuales conforman unidades estructurales y funcionales, definen la cobertura vegetal propia de un lugar (19). Para la región de la Orinoquia 
se encontraron ocho coberturas vegetales (Figura 4). Estas se enumeran y describen a continuación, siguiendo las definiciones propuestas por el Instituto de investigaciones Biológicas Alexander von Humboldt:

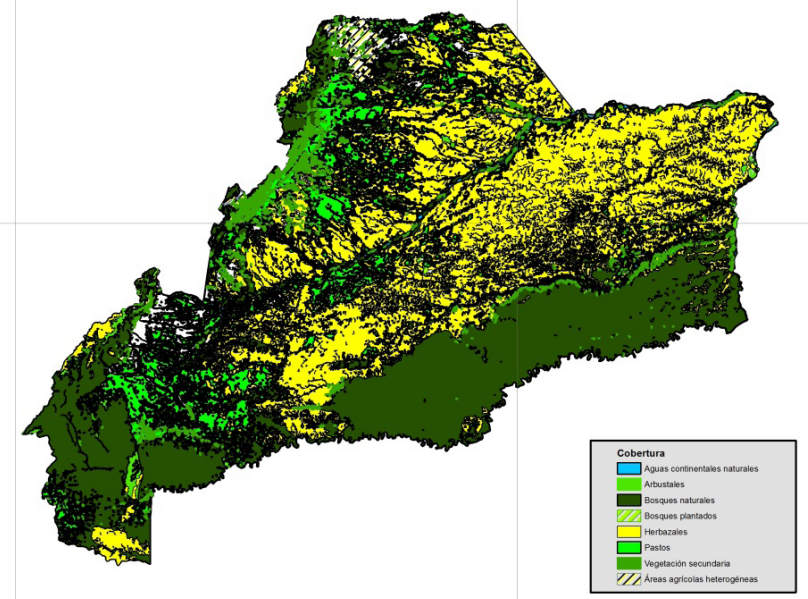

Figura 4. Coberturas vegetales encontradas en la Orinoquia colombiana.

Fuente: Instituto de investigaciones Biológicas Alexander von Humboldt.

Bosques naturales (BN): En esta categoría se encuentran bosques densos, fragmentados, de galería o riparios, y manglares. Esta cobertura se caracteriza por árboles de altura superior a 5 $\mathrm{m}$ y densidad de copas superior al $70 \%$.

Vegetación secundaria (VS): En esta categoría se encuentran rastrojos y cobertura vegetal en estado de sucesión temprano. Esta vegetación se caracteriza por ser de baja altura que generalmente es producto del proceso de sucesión de pastos o cultivos, hacia coberturas arbóreas.

Aguas continentales naturales ( $A C N$ ): Son los ríos, lagunas, lagos o zonas inundadas presentes en el área analizada.

Pastos (PT): En esta categoría se encuentran pastos limpios, arbolados, enmalezados o enrastrojados. Esta cobertura se comprende especies herbáceas que han sido plantadas, generalmente utilizadas para actividades ganaderas.

Herbazales (HBZ): En esta categoría se encuentran herbazales de páramos, de sabanas y xerofíticos, los cuales se caracterizan por la dominancia de hierbas y gramíneas. Adicionalmente, estos herbazales pueden presentar árboles y arbustos.
Áreas agrícolas heterogéneas (AAH): Cobertura que se caracteriza por la combinación de diferentes tipos de cultivos, anuales y permanentes; pastos y cultivos; cultivos, pastos y espacios naturales.

Bosques plantados (BP): Cobertura en donde predominan los bosques plantados de latifoliadas y coníferas.

Arbustales (ABT): Cobertura en la que predomina vegetación leñosa correspondiente a arbustos. Esta categoría incluye arbustales de páramo, de sabana o xerofíticos.

Cultivos anuales o transitorios (CAT): Cobertura vegetal conformada por cultivos con ciclo vegetativo con duración de un año o menos. Generalmente, después de la cosecha, es necesario volver a sembrar o plantar para seguir produciendo.

En la figura 4, se puede observar la distribución de las coberturas vegetales de la región de la Orinoquia. Este mapa se utilizó para el análisis de la intersección entre la orientación ganadera y su asociación con las coberturas vegetales del área de estudio.

Orientación ganadera bovina para la región de la Orinoquia. De acuerdo a la finalidad que tenga el productor ganadero, la orientación ganadera puede clasificarse, grosso modo, en animales especializados para la producción de leche, animales dedicados a la producción de carne y animales de doble propósito. Dentro del grupo dedicado a la producción de carne, este se subdivide en cría, levante y ceba (9). Para la región de la Orinoquia se encontraron 6 orientaciones, las cuales se pueden observar en la Figura 5. Este mapa se utilizó para el análisis de la intersección entre la orientación ganadera y su asociación con las coberturas vegetales, la fisiografía y el catastro del área de estudio.

Catastro para la región de la Orinoquia. Los mapas catastrales son una serie de polígonos que delimitan el área de una propiedad, ya sea de carácter privado, común o del Estado. Para este estudio, corresponde a las áreas ubicadas en la zona rural de la región (Figura 6). Sin embargo, para el departamento del Vichada, solo se encontró el catastro para un tercio de su territorio. 


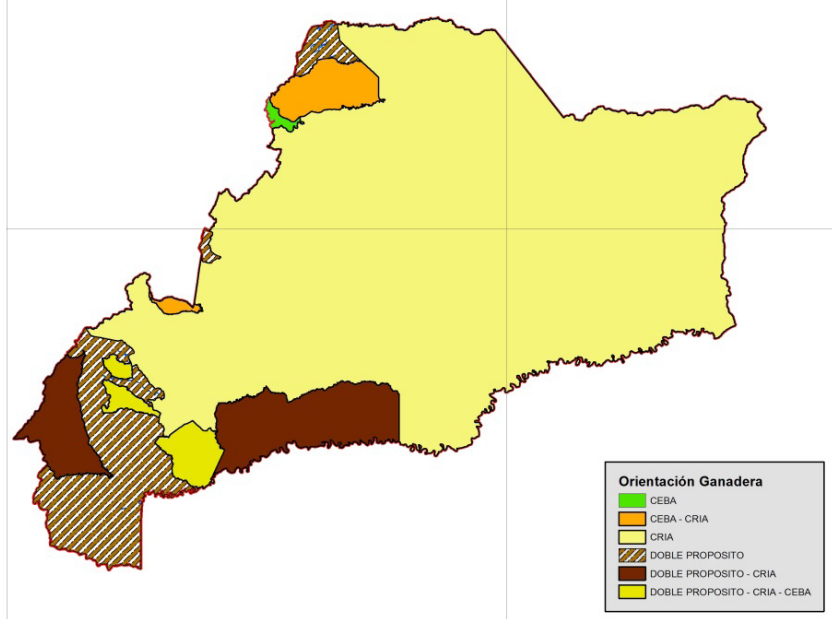

Figura 5. Orientación ganadera de la región de la Orinoquia.

Fuente: Fedegan.

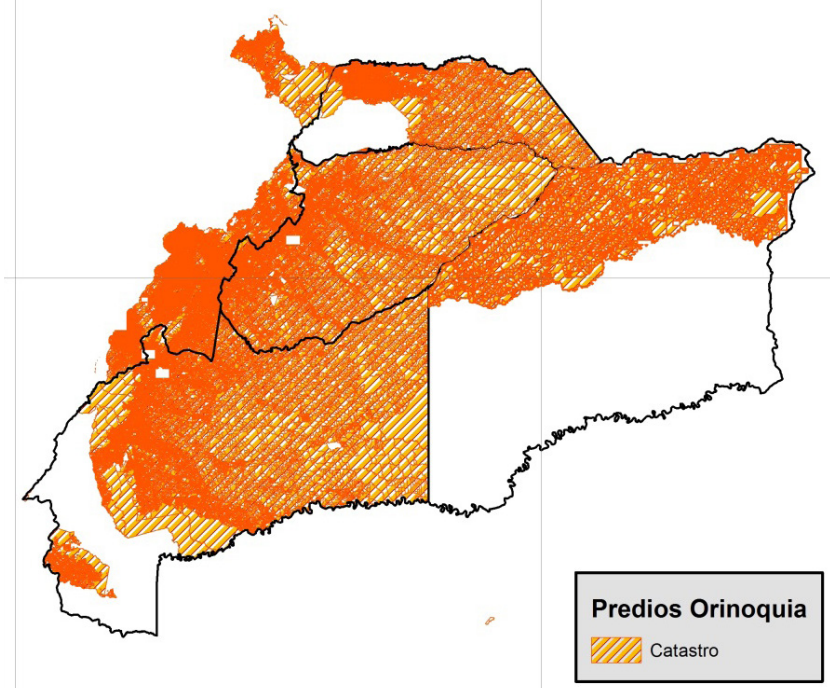

Figura 6. Mapa catastral de la Orinoquia colombiana. Fuente: IGAC

La capa que representaba la división política de la región de la Orinoquia fue utilizada para delimitar el área de estudio, usando la herramienta de geoprocesamiento clip, del software especializado en SIG, ArcGIS versión 10.6. Una vez delimitada la información espacial de acuerdo con las áreas de la región, se realizaron una serie de intersecciones (Intersect), la cual es una técnica de geoprocesamiento de superposición entre dos capas; la primera es la capa de entrada y la otra, se considera de superposición, ambas geometrías deberán ser de tipo polígono y se obtiene como resultado una nueva capa la cual conservará información de ambas (20), generando al final información espacial y tabular, que muestra la combinación entre los parámetros tenidos en cuenta.
Análisis de los datos. Para el análisis de la información se migraron los datos obtenidos en ArcGIS a una interfase especialmente diseñada en Microsoft Access, la cual cuenta con una herramienta de generación de consultas que permite organizar y depurar la información aplicando diferentes filtros según el criterio deseado.

Se elaboraron formularios que permitieron crear diferentes tipos de tablas con información sobre el tipo de cobertura existente, sus proporciones, y predios que cumplieran con diferentes criterios en cuanto a fisiografía, cobertura y orientación ganadera. La principal condición para la inclusión de los predios como ganaderos, teniendo como premisa que más del $95 \%$ de los sistemas ganaderos del país están bajo pastoreo (7), era tener como mínimo cobertura vegetal de pastos, o pastos y diferentes combinaciones con otras coberturas.

Los algoritmos de programación utilizados se elaboraron mediante instrucciones SQL, como DELETE, INSERT INTO, UPDATE, combinando con niveles de agrupación a través de GROUP BY, combinado con SUM, y cuando se requiere, creando ordenamientos, a través de opciones ORDER BY.

Los resultados obtenidos, a partir de la interacción entre SIG y ACCESS, se presentaron de forma descriptiva.

\section{RESULTADOS}

Los resultados de la intersección entre la orientación ganadera, cobertura vegetal, fisiografía y catastro, arrojaron la siguiente información que se presenta para cada uno de los departamentos:

Arauca. Para el departamento de Arauca se encontraron 2576 predios destinados a la ganadería bovina, de los cuales el $98.25 \%$ se orienta a la cría y el $1.74 \%$ al doble propósito. Los 2531 predios dedicados a la cría representan 1094202 ha; los 45 predios dedicados al doble propósito equivalen a 2071 ha.

531 predios, equivalentes a 13100 ha se encuentran solo con coberturas de pastos; 263 predios, equivalentes a 18479 ha se encuentran con coberturas de pastos y bosques naturales; 571 predios, equivalentes a 178516 ha se encuentran con coberturas de pastos y herbazales; 406 predios, equivalentes a 280415 ha se encuentran con coberturas de pastos, herbazales y bosques naturales. 
En la Tabla 1, se muestran las combinaciones de coberturas y fisiografías más recurrentes para cada una de las orientaciones ganaderas en el departamento.

Tabla 1. Combinaciones ganaderas más representativas para el departamento de Arauca.

\begin{tabular}{|c|c|c|c|c|}
\hline Orientación & \multicolumn{4}{|c|}{ Coberturas Fisiografía Predios Área(ha) } \\
\hline Cría & PT, HBZ & PBIAC & 506 & 152188.16 \\
\hline Cría & $\begin{array}{c}\mathrm{PT}, \mathrm{HBZ} \text {, } \\
\mathrm{BN}\end{array}$ & PBIAC & 283 & 177026.67 \\
\hline Cría & PT & PBIAC & 272 & 8348.47 \\
\hline Cría & PT & PDCM & 180 & 2080.06 \\
\hline Cría & PT, BN & PBIAC & 125 & 7982.40 \\
\hline Cría & PT, BN & PDCM & 103 & 4060.02 \\
\hline Cría & $\begin{array}{c}\mathrm{PT}, \mathrm{HBZ} \text {, } \\
\mathrm{BN}\end{array}$ & $\begin{array}{l}\text { PDCM, } \\
\text { PBIAC }\end{array}$ & 97 & 79693.55 \\
\hline Doble propósito & PT, AAH & PBIAC & 19 & 1054.31 \\
\hline Doble propósito & PT, AAH & $\begin{array}{l}\text { PDCM, } \\
\text { PBIAC }\end{array}$ & 12 & 605.16 \\
\hline Doble propósito & $\mathrm{PT}, \mathrm{AAH}$ & PDCM & 12 & 410.31 \\
\hline
\end{tabular}

Casanare. Para el departamento del Casanare se encontraron 23848 predios destinados a la ganadería bovina, de los cuales el $98.74 \%$ se orienta a la cría; el $1.24 \%$ al doble propósito y el $0.0083 \%$ a la ceba. Los 23549 predios dedicados a la cría representan 3009937 ha; los 297 predios dedicados al doble propósito equivalen a 27221 ha y, los 2 predios destinados a la ceba corresponden a 2555 ha.

En la Tabla 2, se muestran las combinaciones de coberturas y fisiografías más recurrentes para cada una de las orientaciones ganaderas en el departamento del Casanare.

Tabla 2. Combinaciones ganaderas más representativas para el departamento del Casanare.

\begin{tabular}{|c|c|c|c|c|}
\hline Orientación & Coberturas & Fisiografía & Predios & Área(ha) \\
\hline Cría & PT & PDCM & 11645 & 74656 \\
\hline Cría & PT, VS & PDCM & 1781 & 81778 \\
\hline Cría & PT, HBZ & PBIAC & 1173 & 329833 \\
\hline Cría & $\mathrm{PT}, \mathrm{HBZ}, \mathrm{BN}$ & PBIAC & 1029 & 527064 \\
\hline Cría & PT & PBIAC & 902 & 33411 \\
\hline Cría & PT, BN & PDCM & 714 & 38684 \\
\hline Doble propósito & PT & PDCM & 68 & 1722 \\
\hline Doble propósito & PT, BN & PDCM & 60 & 5291 \\
\hline Doble propósito & PT, CAT & PDCM & 56 & 1408 \\
\hline Doble propósito & PT, BN, CAT & PDCM & 44 & 3961 \\
\hline Doble propósito & PT, BN, HBZ & PDCM & 13 & 2431 \\
\hline Ceba & $\begin{array}{l}\mathrm{PT}, \mathrm{HBZ}, \mathrm{ABT} \\
\mathrm{BN}, \mathrm{AAH}, \mathrm{CAT}\end{array}$ & VOCO & 1 & 2364 \\
\hline Ceba & $P T, A C N, V S, B N$ & Voco & 1 & 191.55 \\
\hline
\end{tabular}

Rev MVZ Córdoba. 2020. Septiembre-Diciembre; 25(3):e1720 https://doi.org/10.21897/rmvz.1720
13354 predios, equivalentes a 122768 ha se encuentran solo con coberturas de pastos; 2265 predios, equivalentes a 97256 ha se encuentran con coberturas de pastos y vegetación secundaria; 1722 predios, equivalentes a 399100 ha se encuentran con coberturas de pasto y herbazales; 1482 predios, equivalentes a 115161 ha se encuentran con coberturas de pastos y bosques naturales; 312 predios, equivalentes a 42409 ha se encuentran con coberturas de pastos, vegetación secundaria y bosques naturales.

Meta. Para el departamento del Meta se encontraron 31416 predios destinados a la ganadería bovina, de los cuales el $44.16 \%$ se orienta a la cría; el $31.10 \%$ al doble propósito; $18.22 \%$ se orienta al doble propósito-críaceba; el $3.6 \%$ a la ceba-cría y $2.9 \%$ al doble propósito-cría. Los 13874 predios dedicados a la cría representan 373866 ha; los 9759 predios dedicados al doble propósito equivalen a 27221 ha; los 5724 predios destinados al doble propósito-cría-ceba corresponden a 853302 ha; 1136 predios dedicados a la ceba-cría equivalen a 44152 ha y, 923 predios destinados al doble propósito-cría equivalen a 762011 ha.

En la Tabla 3, se muestran las combinaciones de coberturas y fisiografías más recurrentes para cada una de las orientaciones ganaderas en el departamento del Meta.

Tabla 3. Combinaciones ganaderas más representativas para el departamento del Meta.

\begin{tabular}{|c|c|c|c|c|}
\hline Orientación & Coberturas & isiografía & Predios & Área(ha) \\
\hline Cría & PT & VOCO & 5549 & 30133 \\
\hline Cría & PT, CAT & VOCO & 1395 & 64654 \\
\hline Cría & PT, BN & VOCO & 937 & 77693 \\
\hline Cría & $\begin{array}{c}\mathrm{PT}, \mathrm{HBZ} \text {, } \\
\mathrm{BN}\end{array}$ & PAMV & 923 & 956853 \\
\hline Cría & PT & PAMV & 856 & 14539 \\
\hline Doble propósito & PT & PDCM & 2326 & 31218 \\
\hline Doble propósito & PT & voco & 2272 & 22787 \\
\hline Doble propósito & PT & SM & 738 & 10785 \\
\hline $\begin{array}{l}\text { Doble propósito } \\
\text { cría-ceba }\end{array}$ & PT & VOCO & 1133 & 35489 \\
\hline $\begin{array}{l}\text { Doble propósito } \\
\text { cría-ceba }\end{array}$ & $\mathrm{PT}, \mathrm{BN}$ & PDCM & 1002 & 66253 \\
\hline $\begin{array}{l}\text { Doble propósito } \\
\text { cría-ceba }\end{array}$ & PT & PDCM & 764 & 14425 \\
\hline $\begin{array}{l}\text { Doble propósito } \\
\text { cría-ceba }\end{array}$ & PT & PAMV & 764 & 4842 \\
\hline Ceba-cría & PT & VOCO & 519 & 4805 \\
\hline Ceba-cría & $\mathrm{PT}, \mathrm{BN}$ & VOCO & 224 & 13491 \\
\hline $\begin{array}{l}\text { Doble } \\
\text { propósito cría }\end{array}$ & $\mathrm{PT}, \mathrm{BN}$ & PDCM & 144 & 11955 \\
\hline $\begin{array}{c}\text { Doble } \\
\text { propósito-cría }\end{array}$ & $\mathrm{PT}, \mathrm{BN}$ & PAMV & 115 & 28767 \\
\hline $\begin{array}{c}\text { Doble } \\
\text { propósito-cría }\end{array}$ & PT, BN & VOCO & 105 & 14747 \\
\hline
\end{tabular}


Con cobertura de solo pastos se encuentran los siguientes predios: 6885 predios orientados a la cría, representan 62494 ha; 5745 predios orientados al doble propósito, equivalentes a 82726 ha; 2929 predios orientados al doble propósito-cría-ceba, equivalentes a 64314 ha. Con coberturas de pastos más bosques naturales se encuentran los siguientes predios: 2458 predios orientados a la cría, equivalentes a 324042 ha; 1613 predios orientados al doble propósito-cría-ceba, equivalentes a 163330 ha y, 1459 predios orientados al doble propósito que representan 128572 ha. Con coberturas de pastos más herbazales y bosques naturales se encontraron 1043 predios orientados a la cría que totalizan 1059286 ha.

Vichada. Para el departamento del Vichada, de acuerdo a su mapa catastral (shp.), se hallaron 145 predios destinados a la ganadería bovina, de los cuales el $100 \%$ se orienta a la cría. Estos predios representan 3122525 ha.

73 predios, equivalentes a 1091913 ha se encuentran con coberturas de pastos, herbazales y bosques naturales; 46 predios, equivalentes a 289389 ha se encuentran con coberturas de pastos y herbazales.

En la Tabla 4, se muestran las combinaciones de coberturas y fisiografías más recurrentes para cada una de las orientaciones ganaderas en el departamento.

Tabla 4. Combinaciones ganaderas más representativas para el departamento del Vichada.

\begin{tabular}{|c|c|c|c|c|}
\hline Orientació & Cobertura & Fisiografía & Predios & Área (ha) \\
\hline Cría & $\begin{array}{c}\text { PT, BN, } \\
\text { HBZ }\end{array}$ & PBIAC, SM & 43 & 649573 \\
\hline Cría & $\mathrm{PT}, \mathrm{HBZ}$ & $\begin{array}{l}\text { VOCO, } \\
\text { PBIAC }\end{array}$ & 23 & 102824 \\
\hline Cría & $\begin{array}{c}\text { PT, BN, } \\
\text { HBZ }\end{array}$ & $\begin{array}{l}\text { VOCO, } \\
\text { PBIAC }\end{array}$ & 19 & 148140 \\
\hline Cría & $\mathrm{PT}, \mathrm{HBZ}$ & PBIAC, SM & 18 & 136730 \\
\hline
\end{tabular}

\section{DISCUSIÓN}

Muchas veces, para entender los problemas de una actividad en un determinado territorio, es necesario estudiarlos de forma sistémica, articulando las interrelaciones de los componentes. Es aquí donde la caracterización realizada en este estudio, utilizando los sistemas de información geografica, toma relevancia. Generalmente, las caracterizaciones de los sistemas ganaderos se realizan utilizando instrumentos como las encuestas, en donde se recopila información productiva, reproductiva, tecnológica y socioeconómica de la actividad en zonas muy específicas, dado el costo del levantamiento de la información $(6,14,17,21)$. Al utilizar los SIG, como herramienta complementaría para la recopilación de la información, es posible estudiar la actividad ganadera a escalas espaciales mayores, permitiendo tener una visión más amplia del uso y manejo de los recursos naturales a nivel de cuenca, región o departamento, usando información ya existente. Adicionalmente, permite incluir y analizar los diferentes elementos que componen estos agroecosistemas, sus interacciones y su relación con la intervención antrópica, es decir, no solo se caracteriza la actividad por la raza de los animales, inventario ganadero, orientación (leche, carne y doble propósito), parámetros zootécnicos y tamaño del área, si no que permite realizar un análisis espacial incluyendo factores determinantes a la hora de tomar decisiones como son las coberturas vegetales, relieve, suelo, entre otros.

La cantidad de predios ganaderos encontrados en este trabajo, de acuerdo a los polígonos provistos por los Shapefiles catastrales y la condición impuesta para su inclusión en el trabajo, identificó un número de predios para cada departamento que difiere con valores presentados por la Federación Colombiana de Ganaderos «Fedegan» y el Instituto Colombiano Agropecuario «ICA» a través de los ciclos de vacunación de fiebre aftosa para el año 2015. Por ejemplo, para el departamento de Arauca el presente trabajo definió 2576 predios como ganaderos; «Fedegan» e «ICA» encontraron más de 9000. Para el departamento de Casanare, este trabajo definió 23848 predios como ganaderos, «Fedegan»e «ICA» encontraron entre 13 y 14 mil. Para el departamento del Meta, este trabajo definió 31416 predios como ganaderos, «Fedegan»e «ICA» encontraron entre 13 y 14 mil. Para el departamento del Vichada, dada la poca información catastral del shp. con que se trabajó, el número de predios encontrados en este trabajo (145), es muy inferior a los 1,400 predios presentados por «Fedegan» e «ICA». Las diferencias encontradas entre este estudio y los datos de otras entidades podrían atribuirse a que la información de los ciclos de vacunación están supeditadas al número de usuarios que vacunen, asumiendo que el $100 \%$ de los ganaderos del país, o región en 
este caso, lo hagan. Adicionalmente, muchos de los sistemas de producción ganadera del país están conformados por varios predios (polígonos), con registro catastral propio, pero dada su continuidad espacial son manejados como si fueran una sola finca; ante las entidades encargadas de la vacunación solo han registrado un predio.

En un alto porcentaje de los predios ganaderos ubicados en los cuatro departamentos (Casanare: $72.7 \%$, Meta: $49.5 \%$, Arauca: $42 \%$ y Vichada: $32 \%$ ) predominan las coberturas de pastos, herbazales y vegetación secundaria, es decir, confirma la expansión en la frontera agropecuaria que ha tenido la actividad ganadera en el país, haciendo un uso extensivo del suelo $(6,16)$.

En cuanto a la orientación ganadera predominante en la Orinoquía, el $69 \%$ de los predios definidos como ganaderos en este trabajo se encontraban orientados a la cría, $17 \%$ al doble propósito y, el $14 \%$ restante se encontraba distribuido en las otras orientaciones (ceba, ceba-cría, doble propósito-cría, doble propósito-cría-ceba).
Estos resultados concuerdan con los expuestos por $(4,11,12)$, los cuales manifiestan que las características de la región permiten que la orientación ganadera se incline a la producción de carne (cría, ceba, doble propósito) y no a la lechería debido a la ausencia de tecnificación en pastos e infraesructura.

En conclusión, los estudios de caracterización a través de SIG permiten mejorar la planificación y distribución eficiente de los recursos destinados al funcionamiento de los diferentes sistemas productivos, por ejemplo, en zonas donde la matriz de coberturas predominante son los pastizales y herbazales, las estrategias en pro de la sostenibilidad pueden enfocarse en la implementación de sistemas silvopastoriles, contrario a lo que pasaría en zonas donde la matriz tenga un alto porcentaje de bosques naturales.

\section{Conflicto de intereses}

Los autores declaramos que no tenemos conflicto de intereses.

\section{REFERENCIAS}

1. Coppock DL, Fernández $M$, Hiernaux $P$, Huber-Sannwald E, Schloeder C, Corinne $V$, et al. Rangeland Systems in Developing Nations: Conceptual Advances and Societal Implications. En: Briske D, editor. Rangeland Systems Processes, Management and Challenges. Springer: Cham; 2017. URL Disponible en: https://doi.org/10.1007/9783-319-46709-2

2. Sala O, Yahdjian L, Havstad K, Aguiar M. Rangeland Ecosystem Services: Nature's Supply and Humans' Demand. En: Briske $\mathrm{DD}$, Walker LR, editores. Rangeland Systems Processes, Management and Challenges. Springer: Cham; 2017. URL Disponible en: http://link.springer.com/10.1007/978-3319-46709-2

3. Gomez-Casanovas N, Delucia NJ, Bernacchi $\mathrm{CJ}$, Boughton $\mathrm{EH}$, Sparks JP, Chamberlain SD, et al. Grazing alters net ecosystem C fluxes and the global warming potential of a subtropical pasture. Ecol Appl. 2018; 28(2):557-572. https://doi.org/10.1002/ eap. 1670
4. Bustamante C, Rojas L. Reflexiones sobre transiciones ganaderas bovinas en Colombia, desafíos y oportunidades. Biodiversidad en la Práctica. 2018; 3(1):1-29. Disponible en: http://revistas.humboldt.org.co/index.php/ BEP/article/view/516

5. Ruiz D, Martinez J, Figueroa A. Agricultura sostenible en ecosistemas de alta montaña. Biotecnología En El Sector Agropecuario Y Agroindustrial. 2015; 13(1):129-138. URL Disponible en: https://revistas.unicauca. edu.co/index.php/biotecnologia/article/ view/360

6. Bermudez CE, Arenas NE, Moreno-Melo V. Caracterización socio-económica y ambiental en pequeños y medianos predios ganaderos en la región del Sumapaz, Colombia. Rev UDCA Actual Divulg Científica. 2017; 20(1):199-208. URL Disponible en: https:// revistas.udca.edu.co/index.php/ruadc/ article/view/76 
7. Molina RA, Sánchez H, Atzori AS. A conceptual model to describe heat stress in dairy cows from actual to questionable loops. Acta Agron. 2018; 67(1):59-64. http://dx.doi. org/10.15446/acag.v67n1.60612

8. Molina RA, Atzori AS, Campos R, Sanchez H. Using System Thinking to Study Sustainability of Colombian Dairy System. Bus Syst Rev. 2014; 3(2):123-141. Disponible en: http:// www.bslaboratory.net/web/images/BSR/ bsrvol.3issue2-014.pdf

9. Cuenca N, Chavarro F, Díaz O. El Sector De Ganadería Bovina En Colombia. Aplicación De Modelos De Series De Tiempo. Rev la Fac Ciencias Económicas. 2008;16(1):165177. URL Disponible en: https://www. umng.edu.co/documents/63968/69535/ art-11\%282\%29.pdf

10. Molina RA, Sanchez $H$, Campos $R$, Atzori A, Morales JD. Dynamic estimation of greenhouse gas emissions from bovine livestock of Valle del Cauca, Colombia. Acta Agron. 2017; 66(3):422-429. http://dx.doi. org/10.15446/acag.v66n3.58266

11. Vera RR, Hoyos Garcés P. Long-term beef production from pastures established with and without annual crops compared with native savanna in the Eastern Plains of Colombia: A compilation and analysis of onfarm results 1979-2016. Trop GrasslandsForrajes Trop. 2019; 7(1):1-13. URL https:// doi.org/10.17138/tgft(7)1-13

12. Peñuela L, Fernández AP. La ganadería ligada a procesos de conservación en la sabana inundable de la Orinoquia. Orinoquia. 2010; 14(1):5-17. https://orinoquia.unillanos. edu.co/index.php/orinoquia/article/view/87

13. Avellaneda-Torres LM, León Sicard TE, Torres Rojas E. Impact of potato cultivation and cattle farming on physicochemical parameters and enzymatic activities of Neotropical high Andean Páramo ecosystem soils. Sci Total Environ. 2018; 631632:1600-1610. https://doi.org/10.1016/j. scitotenv.2018.03.137

14. Vilaboa AJ, Díaz DP. Caracterización socioeconómica y tecnológica de los sistemas ganaderos en siete municipios del estado de Veracruz , México. Zotecnia Trop. 2009; 27(4):427-436. URL Disponible en: http://www.sian.inia.gob.ve/revistas ci/ ZootecniaTropical/zt2704/pdf/Vol.\%20 27\%20N\%C2\%B0\%204\%20-\%202009.pdf
15. Rao I, Peters $M$, Castro A, SchultzeKraft $R$, White $D$, Fisher $M$, et al. The sustainable intensification of forage-based agricultural systems to improve livelihoods and ecosystem services in the tropics Trop Grasslands-Forrajes Trop. 2015; 3(2):5982. https://doi.org/10.17138/tgft(3)59-82

16. Flórez-Yepes GY, Rincon-Santamaría A, Cardona PS, Alzate-Alvarez AM. Multitemporal analysis of the vegetation cover in the area of influence of the mines located in the high part of Maltería in Manizales, Colombia. DYNA. 2017; 84(201):95-101. http:// dx.doi.org/10.15446/dyna.v84n201.55759

17. Ochoa DK, Valarezo JM. Caracterización y análisis de rentabilidad de los sistemas de producción ganaderos presentes en el cantón Yantzaza, Ecuador. Rev Cedemaz. 2014; 4(1):76-85. Disponible en: https:// revistas.unl.edu.ec/index.php/cedamaz/ article/view/240/

18. Marçal De Carvalho W, De Oliveira Vieira E, Machado J, Rocha J, Kênio A, Pereira S, et al. Caracterização Fisiográfica Da Bacia Hidrográfica Do Córrego Do Malheiro, No Município De Sabará-Mg. Irriga. 2009; 14(3):398-412. https://doi.org/10.15809/ irriga.2009v14n3p398-412

19. Gonzaga C. Aplicación de índices de vegetación derivados de imágenes satelitales para análisis de coberturas vegetales en la provincia de Loja, Ecuador. Cedemaz. 2015; 5(1):30-41. Disponible en: http://revistas. unl.edu.ec/index.php/cedamaz/article/ view/43

20. Almeida P, Duriavich M, Napolitano R, Feoli E. Aplicación de técnicas SIG, Sensoramiento Remoto y Análisis Multicriterio para la Detección de Impactos Antropogénicos en la Cobertura de Suelos y su Proyección para el 2010 . Caso de estudio : Estuario de Santos ( Brasil ). Rev Tecnológica ESPOL. 2009; 22(1):73-79. URL Disponible en: http://www.rte.espol.edu.ec/index.php/ tecnologica/article/view/93

21. Molina RA, Sánchez H. Sostenibilidad de sistemas ganaderos bovinos de alta montaña en Colombia. Rev Investig Agrar y Ambient. 2017; 8(2):29-36. URL Disponible en: http://hemeroteca.unad.edu.co/index. $\mathrm{php} / \mathrm{riaa} / \mathrm{article} / \mathrm{view} / 2028$ 